\title{
The growth of leading-edge distortions on a viscous sheet
}

\author{
L. M. Hocking \\ Department of Mathematics, University College London, Gower Street, London WC1E 6BT, \\ United Kingdom
}

W. R. Debler and K. E. Cook ${ }^{\text {a) }}$

Department of Mechanical Engineering and Engineering Science, University of Michigan, Ann Arbor, Michigan 48109

(Received 7 October 1997; accepted 15 October 1998)

\begin{abstract}
The results of a set of experiments to determine some features of the undulations that develop on the leading edge of a sheet of fluid on an inclined plane are presented. A range of fluid viscosities, fluid volumes, and plate angles was used. In nearly all the cases, the observed disturbances had a triangular or sawtooth shape, with only a single example of a finger or parallel-sided shape appearing. The power-law exponents for the position down the plate of both the tips of the disturbances and their roots, that is, the points where they join the uniform sheet above them, were calculated from a series of photographs, and the corresponding wavelengths measured. The exponents are broadly in line with those that can be deduced from a simple model including viscosity, gravity, and volume flux, and ignoring all capillary effects. This conclusion suggests that the criterion for distinguishing the two types of disturbance does not depend on the global dynamics of the developing structures, and that a detailed analysis of the tip and root regions, where capillarity will be significant, is needed for further progress to be made. (c) 1999 American Institute of Physics. [S1070-6631(99)00402-X]
\end{abstract}

\section{INTRODUCTION}

The leading-edge instability of a sheet of viscous fluid moving under gravity down an inclined plane was first reported by Huppert. ${ }^{1}$ A fixed volume of fluid was spread uniformly across the plane and released. After the leading edge of the fluid had spread a certain distance down the plane it was observed that the edge spontaneously distorted in the spanwise direction. The structure of the distortion developed nonlinearly in two different ways, depending in an unquantified manner on the parameters of the experiment. In one type, fingers of fluid with increasing length appeared, with sides parallel to the lines of greatest slope. These fingers had their roots lying in a line across the plane, above which there was a stationary undivided sheet of fluid. In the other case, the leading edge had a sawtooth shape, with triangles of fluid developing, the tips of the triangles and their bases both moving down the plane, but at different rates, so that the length-to-width ratio of the triangles increased with time. These experiments, and variants of them, have been repeated by a number of other people (Silvi and Dussan, V, Jerrett and de Bruyn $\left.^{3}\right)$. There have also been some numerical simulations (Schwartz ${ }^{4}$.

There are three aspects of these experiments that require theoretical confirmation and explanation. The first concerns the properties of the sheet of fluid before instability. The second is the onset of the instability, both in its location in time (or distance from the point of release), and in its spanwise frequency. The third includes the nonlinear develop-

${ }^{a)}$ Currently at Northwestern University, Department of Biomedical Engineering, 2145 Sheridan Road, Evanston, IL 60208. ment of the instability, the two different types, the criterion for predicting which shape will appear, and the physical cause that distinguishes fingers from triangles.

The first of these three problem areas was partially analyzed by Huppert. ${ }^{1}$ The motion follows from the balance between the component of gravity acting down the plane and the viscous stress on the plane supporting the fluid. The speed, extent, and thickness of the developing sheet can be determined by this balance. To describe the flow in the vicinity of the edge, however, requires the addition of capillarity into the theory, thus introducing two extra parameters, the surface tension, and (for nonwetting fluids) the contact angle. It is also necessary, to complete the specification of the problem, to make allowance for the removal of the contact-line singularity, either by slip, by a cutoff, or by postulating a thin film ahead of the fluid front. For wetting fluids the region near the leading edge was described by Troian et al. ${ }^{5}$ and for nonwetting fluids theoretically by Hocking ${ }^{6}$ and Goodwin and Homsy ${ }^{7}$ and numerically by Moriarty et al. ${ }^{8}$ In a complete description for wetting fluids, van der Waals' forces must be included (de Gennes ${ }^{9}$ and Hocking ${ }^{10}$ ).

Although the motion near the front of the advancing sheet has little effect on the dynamics of the sheet as a whole, it is precisely this part of the whole sheet that seems to play a crucial role in the stability question. The second problem, concerning the linear stability of the leading edge of the sheet, has been studied by many authors: Troian et al. ${ }^{5}$ Hocking, ${ }^{6}$ de Bruyn,${ }^{11}$ Hocking and Miksis, ${ }^{12}$ Fraysee and Homsy, ${ }^{13}$ Lopez et al. ${ }^{14}$ and Spaid and Homsy. ${ }^{15}$ The presence of an instability and a preferred wavelength have been established by these investigations, although the predic- 
tions are weakly (logarithmically) dependent on the small factor which has to be introduced in one way or another to avoid the singularity at the edge. As long as the numerical value of this parameter remains undetermined, precise agreement between theory and experiment cannot be achieved. As far as we know, there has been no attempt to determine, on theoretical grounds, the delay in the appearance of the instability after the instant of release. Huppert ${ }^{1}$ states that the instability occurs when the fluid becomes sufficiently thin. The vertical profile of the sheet before instability contains a "hump" upstream of the leading edge, and it has been widely suggested that the cause of the instability is related to the presence of this hump. Brenner ${ }^{16}$ has shown that, when the normal component of gravity is included in the analysis, the hump disappears when the angle of slope of the plane is reduced. Since this gravity component decreases in importance as the sheet thins, this might explain the delayed appearance of the hump and of the instability. However, Bertozzi and Brenner ${ }^{17}$ have argued that, even when the hump is absent, the flow may be unstable through transient effects.

Our objective here is to study experimentally and theoretically the nonlinear structures developing from the initial instability. These take one or other of two distinct forms, as first described by Huppert, ${ }^{1}$ and remain largely unexplained. Hocking and Miksis ${ }^{7}$ (for a simpler geometry) and Schwartz ${ }^{4}$ have produced some numerical solutions, but these give no clue to the criterion distinguishing the two forms. One possibility is that the triangles are only a temporary feature, and that the angle between the sides of the triangles slowly decreases, so that, given a long enough plate, they would eventually transform into fingers. There is, however, no evidence that such evolution takes place. Another possibility is that there are always, or often, two possible modes of instability, and that which one appears depends on microscopic effects, such as the smoothness of the surface of the plate and the presence of contaminants. However, if random effects can trigger two types of instability, one would anticipate that triangles, for example, might appear on one part of the plate and fingers at another location. As far as we know, the simultaneous appearance of both types has never been observed.

The major contribution to the distinction between the two modes of instability has been made by Silvi and Dussan, $\mathrm{V}^{2}$, who have argued that the distinguishing criterion is the value of the contact angle. They conducted similar experiments to those of Huppert, ${ }^{1}$ but for supporting plates made of two different materials. In their experiments, all the parameters of the problem were held constant, except for the contact angle. They observed fingers when a Plexiglas plate was used and the static contact angle was about $70^{\circ}$, and triangles for a glass plate and a contact angle of about $18^{\circ}$. In motions involving moving contact lines, the dynamic, rather than the static, contact angle is likely to be the more significant. The dynamic contact angle is a function both of static contact angle and the capillary number, which is proportional to the speed of the contact line, and increases as the capillary number is raised. Although Silvi and Dussan V (Ref. 2) only give the values of the static contact angle, their results give some support to a hypothesis that triangles are associated with small dynamic contact angles, and fingers with large ones. When the static angle is very small, the dynamic contact angle is dominated by the capillary effect and large dynamic angles could be achieved by increasing the capillary number. Thus, it might be possible to obtain fingers even for nearly wetting fluids if the capillary number were to be made sufficiently large.

In the search for some understanding of the nonlinear development of the instability, one avenue that has not been explored hitherto is the description of the fundamental structure of the flow in fingers and triangles. Some of the experiments already mentioned have included measurements of the growth rates of these structures. Huppert ${ }^{1}$ reports that the locations of the extreme positions of either type of disturbance were proportional to (time) ${ }^{q}$ for some exponent $q$; for the tips of the fingers the exponent was 0.6 , while the troughs where neighboring fingers join was virtually stationary. For the triangular shapes, the exponents for tips and troughs were 0.35 and 0.28 , respectively. Jerrett and de Bruyn ${ }^{3}$ observed fingers only in their experiments. They report that the average exponent for the tips of the fingers was 0.65 for glycerine (kinematic viscosity $110 \mathrm{cSt}$ ) and 0.52 for a mineral oil (kinematic viscosity $15 \mathrm{cSt}$ ). The troughs showed only a very small downward motion. The slope of the plate was limited in their experiments to values less than $15^{\circ}$, since otherwise the instability did not have sufficient time to develop.

The experiments to be described here were conducted for declinations up to $45^{\circ}$ and for a wide range of viscosities and fluid volumes. They were intended to study the development with time of the nonlinear shapes taken by the leading edge for a wide range of parameters, with the object of obtaining a set of results against which a possible theory could be tested.

\section{EXPERIMENTS}

In our experiments, a fixed volume of fluid was released, in a manner similar to that of Silvi and Dussan, $\mathrm{V},{ }^{2}$ at the topmost portion of the plate, and the fluid volume provided a third variable parameter. The silicone oils used in the experiments had specific densities and nominal kinematic viscosities of $(0.93 ; 10 \mathrm{cSt}),(0.95 ; 20 \mathrm{cSt}),(0.96,50 \mathrm{cSt})$, and $(0.96 ; 100 \mathrm{cSt})$. The results for angles of decline of $10^{\circ}, 15^{\circ}$, $20^{\circ}, 25^{\circ}$, and $45^{\circ}$ are reported here. In most cases, the physical data were taken from the manufacturer's literature, but in the case of the $20 \mathrm{cSt}$ oil the values of the density and viscosity were measured and found to be 0.945 and $19.1 \mathrm{cSt}$, respectively. A Cannon-Fenske tube was used to measure the viscosity; a de Nouy balance gave a value of $0.0228 \mathrm{~N} / \mathrm{m}$ for the surface tension of the $20 \mathrm{cSt}$ oil, which is close to the manufacturer's average value of $0.021 \mathrm{~N} / \mathrm{m}$. The contact angles of the fluids were determined by measuring the static diameter of a microliter drop placed on a glass slide, the volume of the drop being deduced by weighing the slide before and after the drop was placed on it. The drops were allowed to spread for approximately $5 \mathrm{~min}$ before the measurements were taken. The contact angles were found to be quite small, about $2^{\circ}$ for the least viscous fluid and about $5^{\circ}$ 


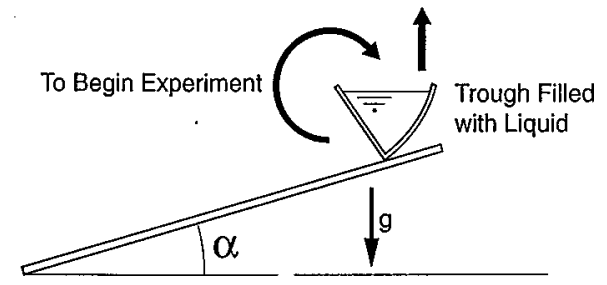

FIG. 1. A sketch of the fluid release mechanism.

for the most viscous. The measurement technique for distilled water produced a value for the contact angle of about $30^{\circ}$.

A glass plate that was somewhat wider than $0.8 \mathrm{~m}$ and twice as long was mounted so that one end could be raised with respect to the other. Millimeter graph paper as well as meter tapes were attached below the plate. These could be viewed and photographed with a $35 \mathrm{~mm}$ camera that was mounted at a fixed distance above the plate, but which could be moved to definite locations along the plate to photograph the film front with a minimum of distortion. A stopwatch was used and the time was recorded for each photograph that was taken. Slide film was used and the transparencies were later projected to measure the position of the film front throughout each experiment.

Prior to starting an experiment, the glass surface was cleaned in a consistent sequence with paper toweling and two solvents, listed as being infinitely soluble by one manufacturer, namely xylene and trichloroethylene. (A second solvent was used in the hope that it would complement the other.) Afterward the angle of decline was set and checks were made with a sensitive spirit level to ensure that the table was not tilted in the lateral direction. The oil was placed in an unattached trough, sealed at each end, with a somewhat $\mathrm{v}$ shaped cross section, as shown in Fig. 1.

One of the edges was convex instead of straight (the piece was actually brass edging for stairs) and this side was placed against the glass. After it was ascertained that the trough was also level, it was slowly rotated along the glass and raised so that the contents spilled out onto the plate toward the upper end. In this way the oil volume, from 40 to $120 \mathrm{~cm}^{3}$, was uniformly distributed across the plate without having any appreciable initial velocity. (In the Silvi and Dussan $\mathrm{V}$ experiments the liquids were contained behind a dam that was raised at the beginning of the experiment. This method was not used by us because some difficulty was experienced in sealing the edge of the relatively long dam that was needed.) While this method of release worked satisfactorily, it may have been less free of disturbances than those used by others, which in turn may have affected the waves that were observed and the time for their initial appearance. In this regard, it should be noted that leading-edge disturbances developed quickly, when the leading edge was still close to the point of release; see Fig. 2. The times and distances associated with photographs in Huppert ${ }^{1}$ and Silvi and Dussan V (Ref. 2) are much greater, but the liquids they used were more viscous.

In all of the experiments reported herein, save one, the disturbance that was observed resembled in planform a tri- angular undulation, a sawtoothed wave. In only one experiment were parallel-sided fingers observed, similar to those illustrated in Huppert ${ }^{1}$ and shown on the cover of the journal in which that article appeared, which were investigated extensively by Jerrett and de Bruyn. ${ }^{2}$ Photographs of the two wave forms are reproduced in Figs. 2 and 3.

Each wave form is characterized by the downplate distances of the tips of the wave, the positions where two teeth or fingers emerge from the continuous film, the roots, and the lateral distance between the tips, the wavelength of the disturbance. Note that in Fig. 2 the shape of the edge is sharply pointed both at the tip and the root, even when the disturbance has become very long. In Fig. 2(c), typical values for the width and length of a triangle are 1.5 and $60 \mathrm{~cm}$, respectively. The fact that the root remained sharp is in sharp contrast to the form of the root regions when fingers are observed, as in Fig. 3, and indicates that the triangular form is not a transient stage on the way to the development of fingers, at least in these instances.

The locations of the roots and tips were obtained from projected images of the slides and stored in a computer for analysis. About 20 roots or tips were observed in each run to obtain the location-time histories. Log-log plots of position versus time were used because of the large time span of each run, and not because of any a priori inference from Huppert's analysis. The graphs of all the data were invariably nearly straight lines. Indeed, if one omitted the first few data points for times, say, under $10 \mathrm{~s}$ for an experiment lasting $200 \mathrm{~s}$ or more, the slope of the line for an individual tip or root was constant with a coefficient of correlation of 0.99 or better in nearly every case. Two typical examples are shown in Fig. 4.

The $40 \mathrm{~cm}^{3}$ experiment produced a finger-like disturbance, and the $80 \mathrm{~cm}^{3}$ one a triangular shape. In both cases only the disturbances close to the center of the plate are included. The straight-line graphs for all the experiments show that the tip and root locations $L$ and the time $t$ are related by laws of the form $L=X t^{q}$, for some positive exponents $q$. The computer was used to obtain a least-squares estimate of the values of these exponents, and these results are given in Table I. The number in parentheses next to the values of the average slopes is the associated standard deviation.

The listings in this table are the average of the statistics for approximately 20 disturbances analyzed for each test. It appears that the distance traveled by the tip along the $45^{\circ}$ decline varies with time in a power law with an exponent slightly greater than $\frac{1}{3}$ and that is practically independent of the viscosity when this is $50 \mathrm{cSt}$ or less. The experiments with $100 \mathrm{cSt}$ oil show a somewhat larger exponent. In contrast to the small differences in the exponent for the tips, that for the roots show more variation, but are generally slightly less than $\frac{1}{3}$. (The data for the roots showed much more variation within an experiment, as indicated by the listed values of the standard deviation.)

Table II displays the wavelengths of the disturbances that occurred for the four liquids at a decline angle of $45^{\circ}$. There appears to be no major change in wavelength for a decade increase in viscosity. 


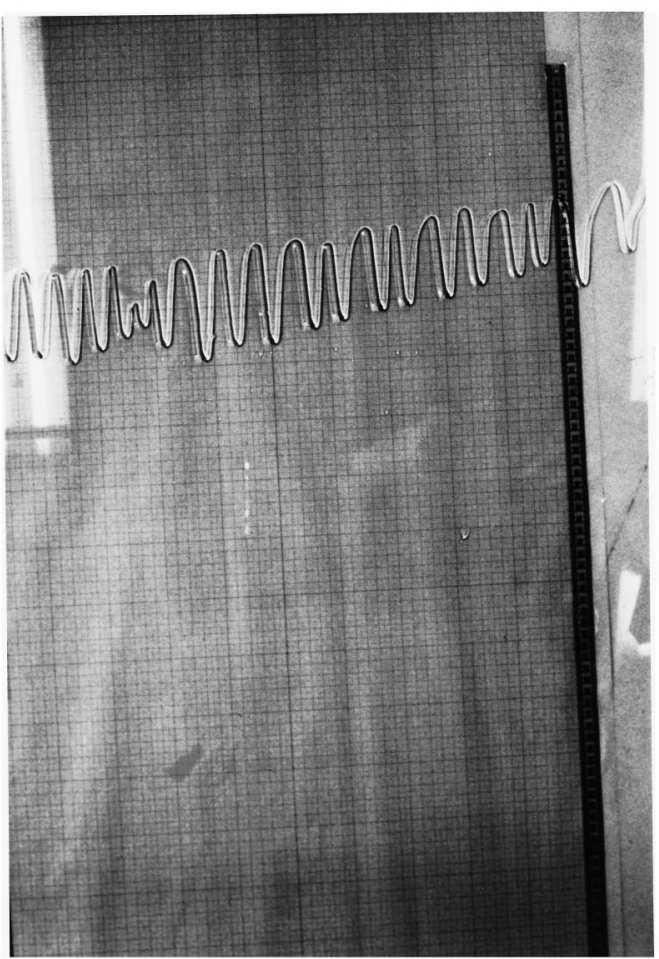

(a)

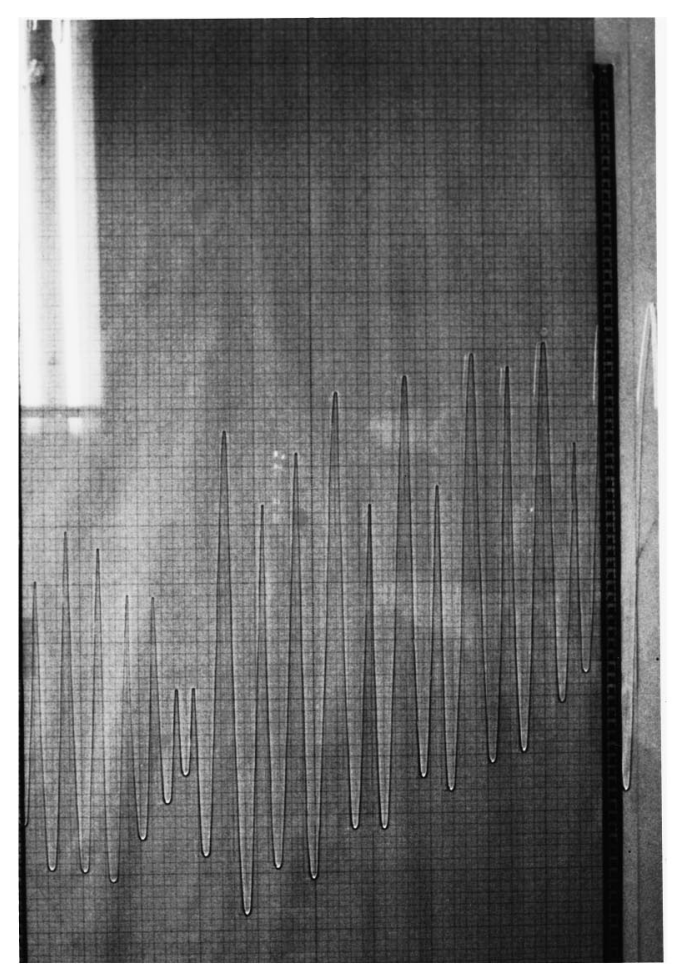

(b)

In Table III the results for the downslope motion are summarized for the (nominal) $20 \mathrm{cSt}$ oil for different volumes of fluid and different slopes. (Accurate values for the properties of this fluid were determined as: kinematic viscosity $19.1 \mathrm{cSt}$, surface tension $22.8 \mathrm{mN} / \mathrm{m}$, density 0.945 $\left.\mathrm{g} / \mathrm{cm}^{3}\right)$. The results suggest no significant variation with angle in the exponents for the tips. The results of Jerrett and

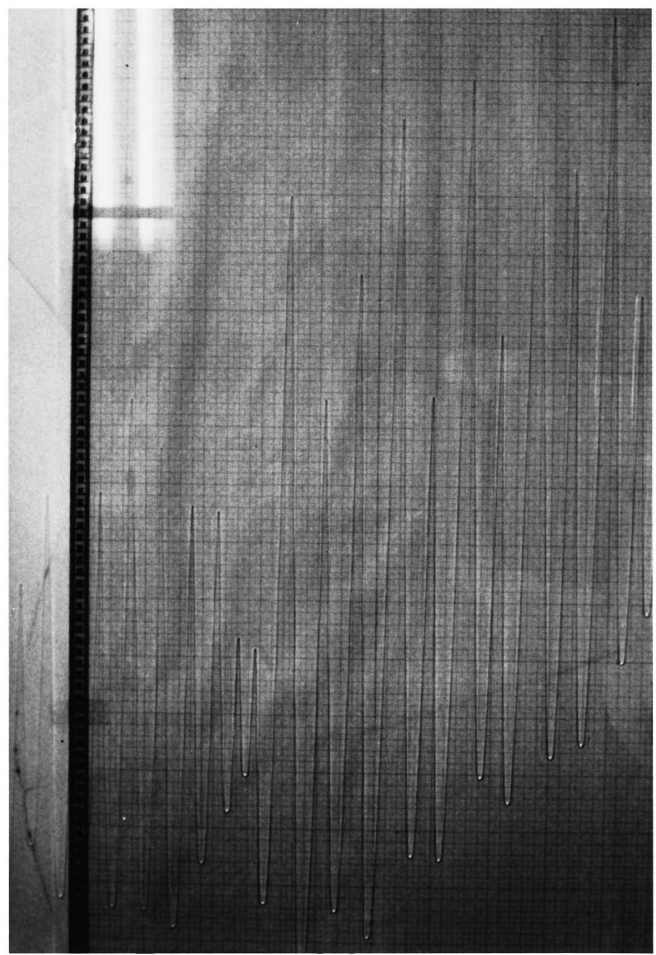

(c)

FIG. 2. The developing triangular shape of the leading edge at increasing times after release: viscosity $20 \mathrm{cSt}$, slope $20^{\circ}$, volume $80 \mathrm{cc}$. The small squares in the background have sides measuring $1 \mathrm{~cm}$. (a) $4 \mathrm{~s}$, (b) $66 \mathrm{~s}$, (c) $307 \mathrm{~s}$.

de Bruyn ${ }^{2}$ similarly show little dependence of the exponent for the finger-like disturbances they observed for angles of decline less than $30^{\circ}$.

Again, the values of the exponents $q$ for the roots have a higher standard deviation than those for the tips. In a few cases, such as for the $25^{\circ}$ slope and $40 \mathrm{~cm}^{3}$ volume, one could note that there was an initial slope of the root graph, 


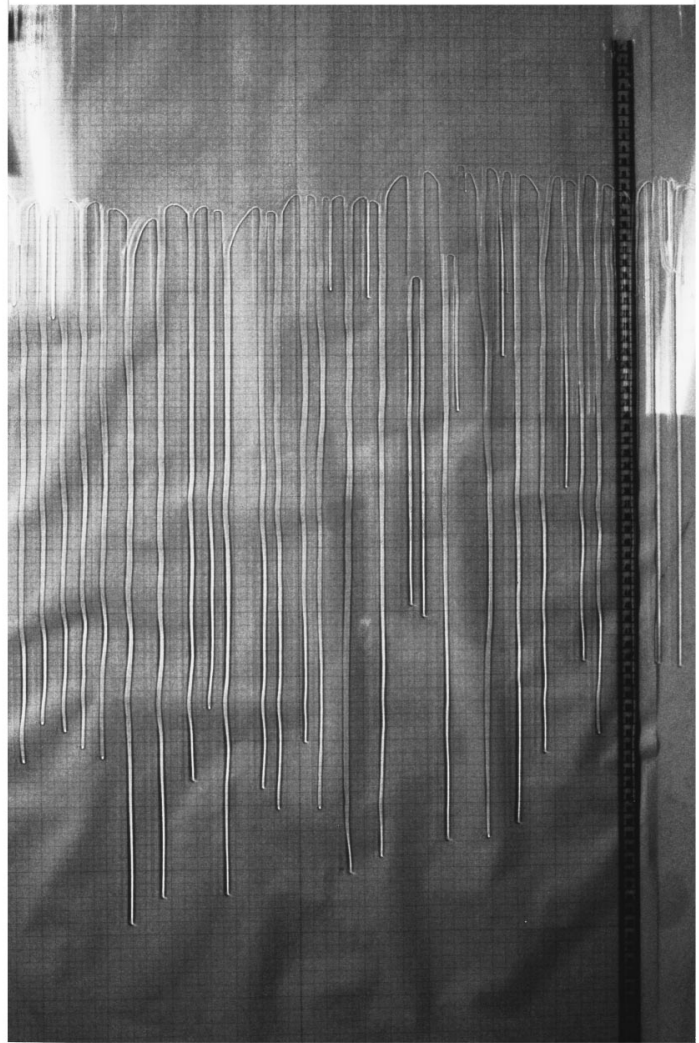

FIG. 3. The finger-like shape of the leading edge at time $179 \mathrm{~s}$ after release: kinematic viscosity $20 \mathrm{cSt}$, slope $45^{\circ}$, volume $40 \mathrm{cc}$. The small squares in the background have sides measuring $1 \mathrm{~cm}$.

which was followed by another straight line of greater slope for the data at later times. This might have resulted from a change in the flow characteristics in the thin film uphill from the front. In addition, the data for the first six roots at $20^{\circ}$ and $80 \mathrm{~cm}^{3}$ have a slope of about 0.16 , but the slope jumps

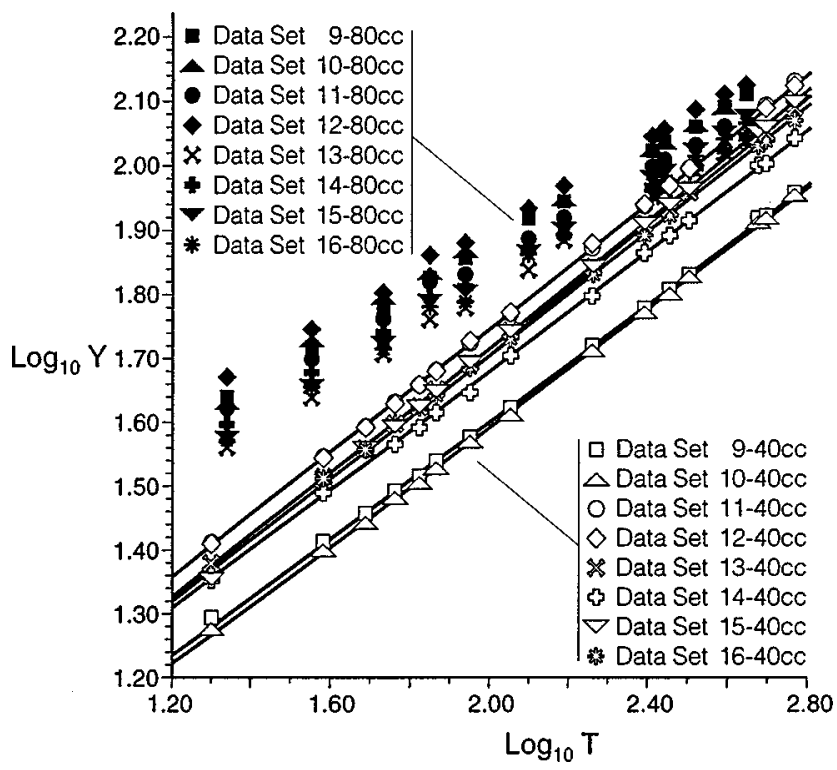

FIG. 4. A plot of distances. $Y$, down a glass plate for disturbances produced when $50 \mathrm{cSt}$ silicone flowed at $45^{\circ}$ to the horizontal direction, as a function of time, $T$.
TABLE I. Tip and root exponents for a $45^{\circ}$ slope. $*=$ fingers. (The numbers in parentheses are the standard deviations.)

\begin{tabular}{cccc}
\hline \hline $\begin{array}{c}\text { Volume, cc } \\
\text { Kinematic viscosity, cSt }\end{array}$ & 40 & 80 & 120 \\
\hline 10, tip & $0.33(0.01)$ & $0.33(0.02)$ & $0.36(0.06)$ \\
10, root & $0.36(0.06)$ & $0.33(0.10)$ & $0.32(0.08)$ \\
20, tip & $0.34(0.01)$ & $0.36(0.02)$ & $0.44(0.02)$ \\
20, root & $0.41(0.07)$ & $0.33(0.09)$ & $0.29(0.09)$ \\
50, tip & $0.49^{*}(0.02)$ & $0.36(0.02)$ & $0.35(0.01)$ \\
50, root & $0.00^{*}$ & $0.24(0.02)$ & $0.28(0.07)$ \\
100, tip & $0.41(0.01)$ & $0.46(0.04)$ & $0.42(0.03)$ \\
100, root & $0.25(0.02)$ & $0.35(0.08)$ & $0.31(0.05)$ \\
\hline \hline
\end{tabular}

abruptly to nearly twice this value for all the other roots. The reason for this is not obvious because the corresponding tip data is very consistent, from which one could infer that the fluid had been placed uniformly across the plate. Note that only the central $80 \%$ of any film was measured so as to minimize any effects of the sidewalls.

Finally, Table IV gives the wavelengths of the sawtooth disturbances as a function of angle and fluid volume for the $20 \mathrm{cSt}$ oil. There is a marked decrease in wavelength as the angle is increased, as also noted by Jerrett and de Bruyn ${ }^{2}$ for the finger-like disturbances.

The capillary number is defined by $\mathrm{Ca}=\mu U / \sigma$, for a fluid with viscosity $\mu$ and surface tension $\sigma$, moving with velocity $U$. Since the velocity of the tips varies throughout each experiment, a range of capillary numbers is covered in each run, with the ratio of high to low values usually lying between 5 and 10 with ratios of over 20 in some cases. Typical examples are provided by the case of $80 \mathrm{~cm}^{3}$ of 50 cSt oil at $45^{\circ}$, for which the largest and smallest capillary numbers were found to be 0.012 and 0.002 , and that of 40 $\mathrm{cm}^{3}$ of $20 \mathrm{cSt}$ oil at $25^{\circ}$, where the capillary numbers decreased from 0.004 to 0.0002 . The extreme values attained by the capillary numbers over all the experiments were 0.012 and 0.0002 . Although there was a large decrease in the capillary number during the course of each experiment, the general pattern of the triangles or fingers, once established, did not change

These experimental results are discussed in the following section, but certain anomalous results are present that are difficult to explain. For example, disturbances of the fingerlike form were observed only for the highest slope and lowest volume, but for a viscosity in the center of the range examined. The range of capillary numbers in this case was from 0.009 to 0.002 , and is similar to that found in all the other cases, in which triangles were formed. Neither the

TABLE II. Wavelengths in $\mathrm{cm}$ for a $45^{\circ}$ slope. $*=$ fingers.

\begin{tabular}{clll}
\hline \hline $\begin{array}{c}\text { Volume, cc } \\
\text { Kinematic viscosity, cSt }\end{array}$ & 40 & 80 & 120 \\
\hline 10 & 2.1 & 2.3 & 2.1 \\
20 & 1.8 & 1.8 & 2.2 \\
50 & $1.8^{*}$ & 2.3 & 2.5 \\
100 & 2.0 & 2.3 & 2.5 \\
\hline \hline
\end{tabular}


TABLE III. Tip and root exponent for $20 \mathrm{cSt}$ silicone oil. *=composite of the first six triangles, averaging $0.16(0.004)$, followed by ten, averaging $0.34(0.06)$ (The numbers in parentheses are the standard deviations.)

\begin{tabular}{lcll}
\hline \hline $\begin{array}{c}\text { Volume, cc } \\
\text { Slope in degrees }\end{array}$ & 40 & \multicolumn{1}{c}{80} & \multicolumn{1}{c}{120} \\
\hline 10, tip & $0.39(0.02)$ & $0.37(0.002)$ & $0.37(0.003)$ \\
10, root & $0.34(0.04)$ & $0.37(0.02)$ & $0.37(0.02)$ \\
15, tip & $0.39(0.03)$ & $0.40(0.02)$ & $0.35(0.004)$ \\
15, root & $0.46(0.02)$ & $0.40(0.06)$ & $0.40(0.02)$ \\
20, tip & $0.36(0.02)$ & $0.35(0.004)$ & $0.36(0.02)$ \\
20, root & $0.39(0.04)$ & $0.27^{*}(0.01)$ & $0.34(0.04)$ \\
25, tip & $0.34(0.02)$ & $0.35(0.01)$ & $0.34(0.004)$ \\
25, root & $0.45(0.03)$ & $0.37(0.03)$ & $0.36(0.05)$ \\
45, tip & $0.34(0.01)$ & $0.36(0.02)$ & $0.44(0.02)$ \\
45, root & $0.41(0.07)$ & $0.32(0.09)$ & $0.29(0.09)$ \\
\hline \hline
\end{tabular}

static nor the dynamic contact angle for the fingers was outside the ranges covered by those for the triangles, which is contradictory to the hypothesis mentioned in the Introduction, that triangles are associated with small contact angles and fingers with large ones. Another peculiarity of the results is that the exponent for the roots sometimes had a value larger than that for the corresponding tips.

\section{GROWTH OF TRIANGLES AND FINGERS}

Theoretical work on the linear stability theory listed in the Introduction assumes that a steady or quasisteady basic state has developed, with a straight leading edge. Small disturbances with a spanwise wavelength are then introduced, and the initial growth rate and preferred spacing calculated. In the experiments described here, there was no such smooth initial state, and the disturbances were present very soon after the fluid was released. In particular, the wavelength cannot be deduced from the linear analysis, since an additional length scale related to the size of the release mechanism may be present. Hence, there is no reason to suppose that the conclusions of linear theory have any relevance to the welldeveloped structures observed in our experiments. Since our objective was to examine the properties of these structures and not their initiation, the lack of a possible comparison with linear theory is not a matter for concern.

In their developed form, both fingers and triangles have a length much greater than their width. Moreover, the Bond number based on their width is given by

$$
B=\frac{\rho g a^{2} \sin \alpha}{\sigma},
$$

TABLE IV. Wavelengths in $\mathrm{cm}$ for $20 \mathrm{cSt}$ silicone oil. (Standard deviations about $0.1 \mathrm{~cm}$.)

\begin{tabular}{cccc}
\hline \hline $\begin{array}{c}\text { Volume, cc } \\
\text { Slope in degrees }\end{array}$ & 40 & 80 & 120 \\
\hline 10 & 3.5 & 3.5 & 4.0 \\
15 & 2.7 & 3.3 & 3.7 \\
20 & 2.5 & 2.7 & 3.4 \\
25 & 2.1 & 2.1 & 2.3 \\
45 & 1.8 & 1.8 & 2.2 \\
\hline \hline
\end{tabular}

where $\rho$ is the density of the fluid, $g$ is gravity, $a$ is the spanwise width, $\alpha$ is the angle of slope of the plate, and $\sigma$ is the surface tension. The value of this parameter in the experiments was at least 10. It follows that the spanwise profile is of near uniform thickness, with capillarity only effective near the edges of the triangle or finger. Hence the finite width of the structure is of little importance in determining the overall dynamics and the basic balance that controls the motion of the fluid in these structures is the same as that in the sheet as a whole, namely, the balance between the downslope component of gravity and the viscous stress on the plate. There is, however, an important difference between them and the preinstability fluid sheet. For the sheet, the volume of the fluid remained constant. In the triangles, however, fluid may continually be fed into each triangle from the fluid that remains above their roots. Thus, the amount of fluid in the triangles increases while the fluid remaining in the sheet decreases. This suggests that the overall dynamics of the triangles can be described by the suggested balance, with a volume constraint that is an increasing function of the time for the triangles themselves, and a decreasing function for the reservoir of fluid. For the fingers, this scheme must be modified. For the evidence suggests that when they appear, the lower edge of the continuous sheet (and the roots of the fingers) remains at rest. Since a static sheet is not possible, an acceptable description of this part of the fluid must allow for the draining of its volume into the top portions of the fingers.

If the height of the fluid measured normal to the plate is small compared with the length and lateral dimension of the triangle or finger, lubrication theory can be used. The balance between gravity and viscous stress gives the differential equation for the height $h$ as a function of the downslope distance $x$ and the time $t$ in the form

$$
3 \mu \frac{\partial h}{\partial t}+\rho g \sin \alpha \frac{\partial h^{3}}{\partial x}=0,
$$

where $\mu$ is the viscosity of the fluid. This equation can be simplified into the form

$$
\frac{\partial h}{\partial t}+\frac{\partial h^{3}}{\partial x}=0,
$$

by writing $\tau=(\rho g \sin \alpha / 3 \mu) t$. A similarity solution of this equation was derived by Huppert ${ }^{1}$ and, more generally, by Lister $^{18}$ to describe the flow of viscous sheets of fluid emerging from a line or point source of variable strength. These solutions have the form

$$
h=\tau^{p-q} f(\eta), \quad \eta=x / \tau^{q}, \quad q=(2 p+1) / 3,
$$

and

$$
(p-1) f-(2 p+1) \frac{d f}{d \eta}+9 f^{2} \frac{d f}{d \eta}=0 .
$$

The length of the sheet is given by $L=X \tau^{q}$ and the volume of fluid in the sheet by

$$
V=\tau^{p} \int_{0}^{X} f(\eta) d \eta .
$$


The constant-volume solution has $p=0, q=\frac{1}{3}$ which is the solution used by Huppert. ${ }^{1}$ For a constant flux of fluid, $p=q=1$ and the sheet has uniform thickness. Values of $p$ between 0 and $-\frac{1}{2}$ and of $q$ between $\frac{1}{3}$ and 0 allow for a decreasing volume of fluid and a leading edge that moves down the plate.

We can now attempt to relate the results of the experiments described in Sec. II to these solutions.

(a) Fingers. Only one set of parameters resulted in the appearance of fingers in these experiments, namely $40 \mathrm{cc}$ volume, $50 \mathrm{cSt}$ kinematic viscosity, and $45^{\circ}$ slope. If the volume of fluid in the finger were constant, the similarity solution would predict that the length of the finger would increase with an exponent equal to $\frac{1}{3}$. The observed exponent is 0.49 , which the similarity solution associates with a volume increase proportional to $t^{0.23}$. This implies that fluid is moving from the reservoir of fluid above the level of the roots of the fingers into the fingers themselves. The roots of the fingers, that is, the lower edge of the sheet between the fingers, appears in the experiments to be at rest. Since the depth of the fluid in the region above this portion of the lower edge is thin, it is possible for its weight to be supported by surface tension, and consequently be stationary (the similarity solution only applies when surface tension plays an insignificant role). Note that, in the experiments for small angles reported by Jerrett and de Bruyn, ${ }^{3}$ the average exponents for fingers are 0.65 (kinematic viscosity $110 \mathrm{cSt}$ ), and 0.52 (kinematic viscosity $15 \mathrm{cSt}$ ).

(b) Triangles. The exponents for the growth of the lengths of the triangles varies in the experiments between 0.33 , which corresponds to a triangle of constant volume, to 0.46 , for which the similarity solution indicates that the volume is proportional to $t^{0.19}$. The increase in volume must come from a draining of the fluid in the upper portion above the roots into the triangles. The roots of the triangles, in contrast to those of the fingers, move down the plane with a range of exponents from 0.25 to 0.46 , and sometimes the exponent for the root is greater than that for the tip of the triangle. However, in all cases the length of the triangle between tip and root is increasing. For the fingers, the roots extend over a region comparable in width to that of the fingers themselves, while the triangles span the whole width of the plate and the roots, like the tips, have no spanwise extent. Consider the whole region comprising one triangle and the region above its base, and suppose strict periodicity. The volume of the fluid in this region must be constant, and the similarity solution would indicate that both tip and root should move downward with the positions of both proportional to $t^{1 / 3}$. Although some of the results shown in Tables I and III fit this prediction, others do not. If there is a transfer of fluid from the upper region into the triangles, as the results suggest, then the similarity solution is not appropriate for the upper region, nor for the positions of the roots of the triangles. It seems likely that, as for the fingers, a satisfactory analysis of the region where the triangles join the upper sheet of fluid, including the regions where two triangles meet, requires the inclusion of surface tension as a significant contributor to the dynamics of the motion. However, for both fingers and triangles, the growth of these features, as ob- served in the experiments reported here, is not inconsistent with the similarity solution of Huppert ${ }^{1}$ and Lister, ${ }^{18}$ which is based on the balance between gravity and viscous stresses only.

\section{CONCLUSIONS}

The comparison of these theoretical considerations and the results of the experiments suggests that the overall dynamics of both fingers and triangles is the same as that for a uniform sheet, with constant or changing volume. It follows that the simple model for the motion used here is not adequate to indicate the difference between the two types of structures that occur. To make further progress in the search for a reason for the duality, the regions where surface tension is significant will have to be examined, the edges of the triangles and fingers, their tips, and also the root regions, where two neighboring features and the undivided portion of the sheet of fluid join.

\section{ACKNOWLEDGMENTS}

This work was partially supported by a grant for collaborative research from NATO. Oil samples were kindly supplied, by the Dow Corning Corporation. We are pleased to record our thanks to Christina Degnore, whose reliable assistance in many phases of the experiments contributed to the good results that were obtained.

${ }^{1}$ H. E. Huppert, "Flow and instability of a viscous current down a slope," Nature (London) 300, 427 (1982).

${ }^{2}$ N. Silvi and E. B. Dussan V, "On the rewetting of an inclined solid surface by a liquid," Phys. Fluids 28, 5 (1985).

${ }^{3}$ J. M. Jerrett and J. R. de Bruyn, "Finger instability of a gravitationally driven contact line,', Phys. Fluids A 4, 234 (1992).

${ }^{4}$ L. W. Schwartz, "Viscous flows down an inclined plane: Instability and finger formation," Phys. Fluids A 1, 443 (1989).

${ }^{5}$ S. M. Troian, E. Herbolzheimer, S. A. Safran, and J. F. Joanny, "Fingering instabilities of driven spreading films," Europhys. Lett. 10, 25 (1989).

${ }^{6}$ L. M. Hocking, "Spreading and instability of a viscous fluid sheet," J. Fluid Mech. 221, 373 (1990).

${ }^{7}$ R. Goodwin and G. M. Homsy, "Viscous flow down a slope in the vicinity of a contact line," Phys. Fluids A 3, 515 (1991).

${ }^{8}$ J. A. Moriarty, L. W. Schwartz, and E. O. Tuck, "Unsteady spreading of thin liquid films with small surface tension," Phys. Fluids A 3, 773 (1991).

${ }^{9}$ P. G. de Gennes, 'Wetting: statics and dynamics," Rev. Mod. Phys. 57, 827 (1985).

${ }^{10}$ L. M. Hocking, "The wetting of a plane surface by a fluid," Phys. Fluids 7, 1214 (1995).

${ }^{11}$ J. R. de Bruyn, "Growth of fingers at a driven three-phase contact line," Phys. Rev. A 46, 4500 (1992).

${ }^{12}$ L. M. Hocking and M. J. Miksis, "Stability of a ridge of fluid," J. Fluid Mech. 247, 157 (1993).

${ }^{13}$ N. Fraysee and G. M. Homsy, "An experimental study of rivulet instabilities in centrifugal spin coating of viscous Newtonian and nonNewtonian fluids," Phys. Fluids 6, 1491 (1994).

${ }^{14}$ P. G. Lopez, S. G. Bankoff, and M. J. Miksis, "Non-isothermal spreading of a thin liquid film on an inclined plane,"' J. Fluid Mech. 324, 261 (1996).

${ }^{15}$ M. A. Spaid and G. M. Homsy, "Stability of Newtonian and viscoelastic dynamic contact lines,' Phys. Fluids 8, 460 (1996).

${ }^{16}$ M. P. Brenner, "Instability mechanisms at driven contact lines," Phys. Rev. E 47, 4597 (1993).

${ }^{17}$ A. L. Bertozzi and M. P. Brenner, "Linear stability and transient growth in driven contact lines," Phys. Fluids 9, 530 (1997).

${ }^{18}$ J. R. Lister, "Viscous flows down an inclined plane from point and line sources," J. Fluid Mech. 242, 631 (1992). 